\title{
EVALUATION OF IMMUNOCHROMATOGRAPIC TEST USING Tp17 ANTIGEN FOR DETECTION OF TREPONEMAL ANTIBODY IN BLOOD DONORS
}

\author{
Dwi Rahayuningsih", Aryati ${ }^{1}$, Budi Arifah ${ }^{2}$ \\ Department of Clinical Pathology, Faculty of Medicine, Airlangga University/Dr. Soetomo Hospital, Surabaya, Indonesia. E-mail: \\ dr_aryati@yahoo.com; dwi.rahayuningsih84@gmail.com \\ Blood Transfusion Unit of Surabaya Indonesian Red Cross, Indonesiadr_aryati@yahoo.com
}

\begin{abstract}
Syphilis transmission through blood transfusion urged the WHO to recommend a detection of treponemal antibody in blood donors. The treponemal antibody was identified to be formed against the membrane of lipoprotein antigen Tp15, Tp17, and Tp47 of T.pallidum. The Tp17 antigen may have an important role in the pathogenesis of syphilis. Evaluation of the CLIA method using Tp17 antigen showed a good diagnostic value. Currently, the immunochromatography test using Tp17 antigen is available but the diagnostic value has not been widely published. The aim of this study was to determine the diagnostic value of immunochromatographic test using Tp17 antigen for treponemal antibody detection in blood donors. A total of 100 serum samples with reactive $(n=66)$ and non-reactive $(n=34)$ treponemal antibody were screened by using ELISA and CLIA methods in the Blood Transfusion Units of Surabaya, Mojokerto, and Sidoarjo Indonesian Red Cross from May 2018-August 2018 and were examined for treponemal antibody with immunochromatographic test using Tp17 antigen (StandardTM Q Syphilis Ab, Standard Biosensor) and Fluorescent Treponemal Antibody Absorption /FTA-ABS (EUROIMMUN, AG) as the gold standard. Kappa Cohen analysis showed that there was moderate and significant concordance between immunochromatographic test using Tp17 and IgG anti-treponemal FTA-ABS $(k=0.477$ p: 0.000$)$. The anti-treponemal IgM was non-reactive in all samples with the sensitivity and specificity of $69.8 \%$ and $81 \%$, respectively. The low sensitivity was possibly due to the use of a single antigen (Tp17) while the treponemal antibody was formed by Tp15, Tp17, and predominantly by Tp47 antigen. The others possibilities were the decreased anti-Tp17 IgG in donors after syphilis treatment and the use of different gold standard from other studies (FTA-ABS vs TPHA). Further study using routine TPHA as a confirmation test, Western Blot to determine the other antibodies than anti-Tp17, and non-treponemal test to determine the disease activity was needed.
\end{abstract}

Key words: Syphilis, immunochromatographic test, Tp17 antigen, treponemal antibody

\section{INTRODUCTION}

The risk of syphilis transmission through blood transfusion urged WHO to recommend a detection of treponemal antibody in blood donors. ${ }^{1}$ The treponemal test includes several methods such as fluorescent treponemal antibody absorption (FTA-ABS), Treponema pallidum particle agglutination (TPPA), Treponema pallidum hemagglutination (TPHA), Enzyme-Linked Immunoassay (ELISA), Chemiluminescent Immunoassay (CLIA), and immunochromatographic test. ${ }^{2,3}$

Pusdatin data by The Indonesian Ministry of Health in 2014 showed that immunochromatographic test was still the most widely used method for screening transfusion-transmitted infections (TTIs) in the Blood Transfusion Unit (BTU) of the Indonesian Red Cross throughout Indonesia. ${ }^{4}$ The regulation of ministry of Health article 83 of 2014 stated that immunochromatographic tests were mainly used in first class of BTU, although it could still be used for a middle class $B T{ }^{5}$

Immunochromatographic tests are simple and profitable methods because they do not require special laboratory equipment, special skills, they are easy and quite affordable. Another advantage is that it requires relatively easy storage (storage at room temperature/without a refrigerator) and the test's results are relatively fast, although this method is not suitable for use in large scale because it cannot be automated. Immunochromatographic tests with good validity significantly contribute to blood screening tests in areas with relatively small test volumes ( $<20$ tests/day). ${ }^{1}$

The treponemal antibody was identified to be produced against Tp15, Tp17, and Tp47 lipoprotein 
membrane antigen of T.pallidum. ${ }^{6}$ These three antigens were most frequently used for the treponemal antibody test. The Tp17 antigen in several studies was mentioned to have an important role in binding protein ligands, maintaining bacterial membrane structure, and in the pathogenesis of syphilis. ${ }^{6}$ The Tp17 antigen is a strong immunogen that plays an important role in the pathogenesis of syphilis. ${ }^{7}$ A study by Sato et al. using the immuno-slot blot method found that the strongest reactivity was against Tp17 antigen compared to other antigens. ${ }^{8}$

The Tp17 antigen using CLIA (Immulite 1000) method has a sensitivity of $100 \%$ and specificity of 98.8\% to TPHA. ${ }^{9}$ LIASON CLIA using Tp17 antigen has a sensitivity of $95.2 \%$ in primary and secondary syphilis. ${ }^{10}$ Immunochromatographic test using Tp17 antigen is now available; however, the diagnostic value has not been widely published until now.

Fluorescent Treponemal Antibody Absorption (FTA-ABS) is still considered as the gold standard for syphilis serologic testing because of its high sensitivity among other tests. FTA-ABS detects treponemal antibody at the earliest stage of syphilis. ${ }^{2,3}$ FTA-ABS detects anti-treponemal IgM and IgG. Anti-treponemal IgM can indicate an early-stage infection, while anti-treponemal IgG can indicate an exposure to T.pallidum infection in all stages of treated or untreated syphilis. ${ }^{11}$ FTA-ABS test of anti-treponemal IgM and IgG as the gold standard in this study was expected to screen all donors with treponemal antibody at all stages.

The aim of this study was to evaluate immunochromatographic tests using Tp17 antigen for detection of treponemal antibody in blood donors compared to FTA-ABS test of anti-treponemal IgM and IgG as the gold standard.

\section{METHODS}

The study was conducted from May to August 2018 using a cross-sectional design. Based on the samples size formula, a minimum of 86 samples (confidence interval 95\% and significant of 0.05 ) was required. Samples were taken consecutively from the remaining serum that had been screened for TTIs by ELISA and CLIA methods at the BTUs of Indonesian Red Cross in the city of Surabaya, Mojokerto, and Sidoarjo. Samples that met the inclusion criteria such as samples with reactive treponemal antibody and non-reactive treponemal antibody (samples with reactive $\mathrm{HBsAg}$, reactive anti-HCV, reactive anti-HIV, and non-reactive TTIs) were separated in two aliquots for storage at $-80^{\circ} \mathrm{C}$ before test. Visually lipemic, icteric, and lysis samples were excluded from this study.
A total of 100 serum samples with reactive $(n=66)$ and non-reactive treponemal antibody $(n=34)$ based on the ELISA and CLIA methods were obtained at the BTU of Indonesian Red Cross. 40 samples with non- reactive treponemal antibody consisted of 14 samples (41.2\%) with reactive $\mathrm{HBsAg}$, 7 samples (20.5\%) with reactive anti-HCV, 4 samples (11.8\%) with reactive anti-HIV, and 9 samples (26.5\%) without reactive TTIs. All samples were tested for treponemal antibody by immunochromatography using Tp17 antigen (StandardTM Q Syphilis Ab, Standard Biosensor), with FTA-ABS test of anti-treponemal IgM and IgG (EUROIMMUN AG) as the gold standard.

The immunochromatographic test used Tp17 antigen and procedure manual of FTA-ABS (anti-treponemal IgM and IgG) test following the manufacturer's instructions. Treponemal antibody test results by immunochromatographic test using Tp17 antigen and FTA-ABS were presented as qualitative data (reactive or non-reactive) and consecutively interpreted by three people. Treponemal antibody detection with immunochromatographic test using Tp17 antigen was carried out at the Clinical Pathology Laboratory of the Dr. Soetomo Hospital, Surabaya. FTA-ABS of anti-treponemal IgM and IgG was carried out at the Clinical Pathology Laboratory of the Dr. Cipto Mangunkusumo Hospital, Jakarta.

This research had been approved by the Health Research Ethics Committee of the Faculty of Medicine, Airlangga University, Surabaya Indonesia, with ethical conduct certificates number 198/EC/KEPK/FKUA/2018.

Immunochromatographic test using Tp17 antigen was evaluated by calculating diagnostic values such as sensitivity, specificity, positive predictive value, negative predictive value, and accuracy using a $2 \times 2$ table and comparing the results to the FTA-ABS test of anti-treponemal IgM and IgG as the gold standard. Cohen's Kappa analysis was performed to assess the concordance between immunochromatographic test using Tp17 antigen and FTA-ABS of anti-treponemal IgM and IgG $(p<0.05$ was considered as significant).

\section{RESULTS AND DISCUSSION}

FTA-ABS test of 100 samples showed 63 (63\%) samples with reactive anti-treponemal IgG and 37 (37\%) samples with non-reactive anti-treponemal IgG. All samples showed non-reactive anti-treponemal IgM (Figure 1). 


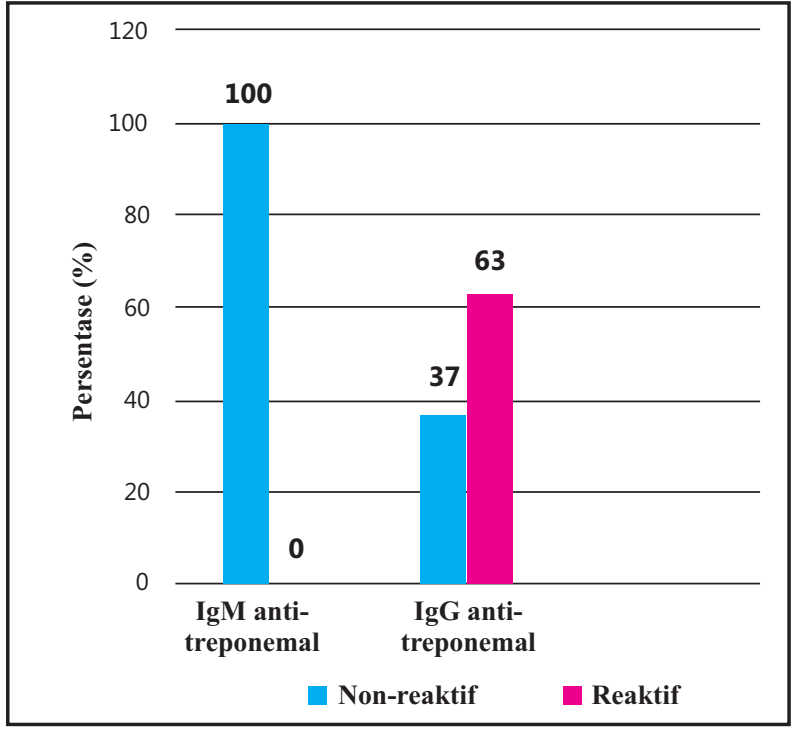

Figure 1. Results of antitreponemal IgM and IgG by FTA-ABS

Reactive anti-treponemal IgG shows exposure to T.pallidum. Anti-treponemal IgG can be reactive for life in syphilis patients with or without treatment, whereas anti-treponemal IgM indicates early-stage infection. ${ }^{11,12}$ Sixty-three samples with reactive anti-treponemal IgG result and non-reactive anti-treponemal IgM showed probability of T.pallidum infections at later stage. Stage and disease progression can be determined by confirmation of clinical history and non-treponemal test (RPR or VDRL). ${ }^{11}$

The results of treponemal antibody detection by immunochromatographic test using Tp17 antigen in 100 samples showed 51 (51\%) samples with reactive treponemal antibody and 49 (49\%) samples with non-reactive treponemal antibody (Figure 2).

The results of treponemal antibody detection with immunochromatographic test using Tp17 antigen compared to FTA-ABS test of anti-treponemal IgG were presented in Table 1 . Analysis of anti-treponemal IgM was not carried out because anti-treponemal IgM was non-reactive in all 100 samples in this study.

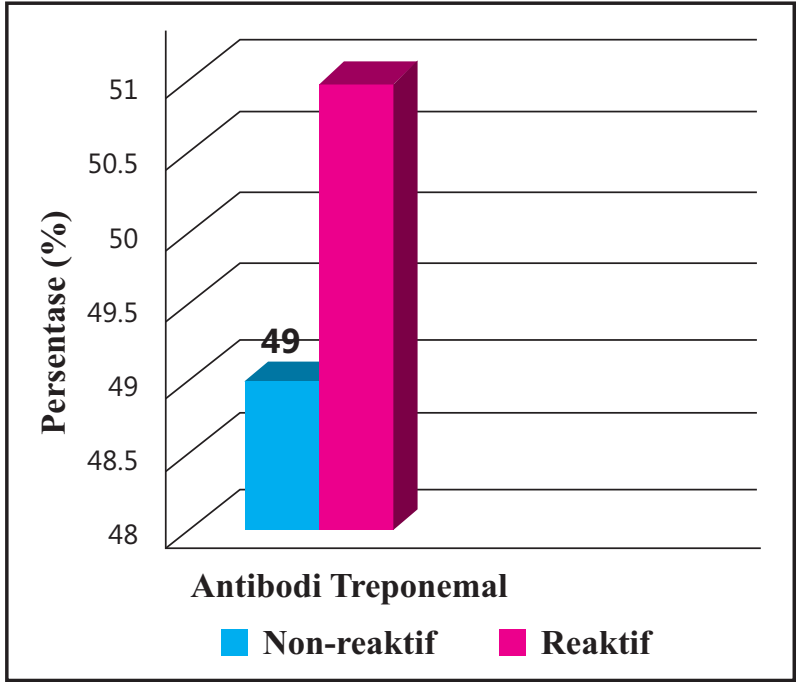

Figure 2. Results of treponemal antibody detection by immunochromatographic test using Tp17 antigen

The results of the analysis showed 19 samples with false negative results. False negative results may be due to the single use of antigen (Tp17) in immunochromatographic test, while the treponemal antibody was identified to be formed against the Tp15, Tp17, and Tp47 antigen of T.pallidum. ${ }^{2,6,12-16}$ The causes of false negative results can be confirmed by treponemal antibody analysis using the Western Blot method to detect treponemal antibody to other antigen than Tp17 which was probably not detected by the immunochromatographic test used in this study.

Ran et al. stated that anti-treponemal IgG decreased after therapy. ${ }^{17}$ This might also be the cause of false negative results in this study with the possibility that 19 samples with false negative results might have come from healthy donors with a history of syphilis and its treatment. Clinical data or history of donor and non-treponemal antibody tests such as VDRL or RPR were needed to confirm these results.

A decrease or not detected anti-Tp17 in donors with a history of syphilis therapy likely revealed the potential of anti-Tp17 as a serological test that can

Table 1. Results of treponemal antibody detection by immunochromatographic test using Tp17 antigen compared with FTA-ABS test of anti-treponemal IgG

\begin{tabular}{clccc}
\hline & & \multicolumn{2}{c}{$\begin{array}{c}\text { FTA-ABS Test of Anti } \\
\text { treponemal IgG }\end{array}$} & \multirow{2}{*}{$\begin{array}{c}\text { Total } \\
(\mathbf{n = 1 0 0 )}\end{array}$} \\
\cline { 3 - 4 } & & Positive & Negative & \\
\hline Immunochromatographic & Positive & 44 & 7 & 51 \\
test using Tp17 antigen & Negative & 19 & 30 & 49 \\
& & 63 & 37 & 100 \\
\hline
\end{tabular}


be used to monitor therapy for syphilis patients.

19 samples with false negative results consisted of 11 samples $(57.9 \%)$ with reactive treponemal antibody, 4 samples (21.1\%) with reactive HBsAg, 3 samples (15.8\%) with reactive anti-HIV, and 1 sample (5.3\%) with normal results detected by ELISA/CLIA at BTU of Indonesian Red Cross. 18 samples with false negative results detected by the immunochromatographic test using Tp17 antigen might not be too problematic because the BTU of Indonesian Red Cross screening test on the sample showed reactive HBsAg and anti-HIV so that blood was excluded. One sample with false negative result by immunochromatographic test using Tp17 antigen and the non-reactive result by ELISA/CLIA was proceeded with VDRL test to determine the disease progression because the sample passed the TTIs screening at the BTU while the FTA-ABS test showed reactive anti-treponemal IgG and non-reactive anti-treponemal IgM. The result of VDRL test was non-reactive, suggesting the possibility of exposure to previous syphilis infection that had been treated. ${ }^{15}$ Clinical data and history of donor were needed to confirm this. Another possibility that caused false positive results of FTA-ABS test was SLE. ${ }^{10,16,18}$

Blood with a reactive treponemal antibody that passed the screening test were relatively safe because the risk of syphilis transmission through blood donors was presumed to decrease when blood products were stored at $4^{\circ} \mathrm{C}$ for more than 2-5 days. The T.pallidum were reported to be resistant to extreme temperatures. ${ }^{19}$ The bag of Thrombocyte Concentrate (TC) was also designed to provide $\pm 15 \%$ oxygen flow. This higher oxygen concentration did not allow T.pallidum to survive (the oxygen concentration required by T.pallidum is $1 \%-4 \%)$. The T.pallidum was also reported to be attached to the surface of phagocytes and the use of leukoreduction blood component was reported to reduce syphilis transmission due to its ability to filter and remove leukocytes from the blood component product. $^{20}$

Seven samples with false positive results consisted of 6 samples (85.7\%) with reactive treponemal antibodies and 1 sample (14.3\%) with the normal result as detected by ELISA/CLIA at the BTU of Indonesian Red Cross. It was stated in some references that the false positive results of the treponemal test could be caused by several conditions such as bacterial/viral infections, liver/hepatitis disease, narcotics usage, SLE/other autoimmune diseases, rheumatoid arthritis, and diabetes. ${ }^{20}$ Based on to the results of ELISA and CLIA at the BTU, viral hepatitis infection was not identified in these seven samples.

Cohen's Kappa analysis showed a moderate and significant concordance between immunochromatographic test using Tp17 antigen and FTA-ABS of anti-treponemal IgG ( $k=0.477$ $\mathrm{p}:$ 0.000). The diagnostic value of the immunochromatographic test using Tp17 antigen showed a sensitivity, specificity, positive predictive value, negative predictive value, accuracy, positive likelihood ratio, and negative likelihood ratio of $69.8 \%, 81 \%, 86.3 \%, 61.2 \%, 74 \%, 3.69$, and 0.37 , respectively.

There were difference of sensitivity and specificity between the immunochromatographic test using Tp17 antigen and CLIA method using Tp17 antigen (Immulite 1000). CLIA method using Tp17 antigen had a sensitivity and specificity to TPHA of $100 \%$ and 98.8\%, respectively. 'LIASON CLIA using Tp17 antigen showed a sensitivity of $95.2 \%$ in primary and secondary syphilis, but its diagnostic value for latent or post-therapy syphilis was not mentioned. ${ }^{10}$

Discrepancy in results could be caused by the use of different methods (immunochromatography or CLIA) or could also be caused by the use of different confirmation tests (TPHA or FTA-ABS). TPHA was developed in the same era as FTA-ABS, but to date FTA-ABS is still considered as the gold standard for confirmation of syphilis serological tests based on the detection of treponemal antibody. ${ }^{10,16,18}$

FTA-ABS detects specific anti-treponemal IgM and IgG of the T. pallidum protein. This test uses the principle of indirect immunofluorescence using the antigen of T.pallidum subspecies pallidum (Nichols strain). ${ }^{10,16,18}$ TPHA uses the principle of hemagglutination by the antigen of T.pallidum subspecies pallidum (Nichols strain) but detects total treponemal antibody. It was unclear whether the use of different antibody detection (total or anti-treponemal IgM and IgG) were the cause for different results. The sensitivity of FTA-ABS in the primary infection, secondary infection and early latency, further latent infection was $84 \%, 100 \%$, and $96 \%$, with the specificity of $97 \%$, respectively. The sensitivity of TPHA at the primary infection, secondary infection, latent infection, and further latent infection was $76 \%, 100 \%, 97-100 \%$, 94\%, with the specificity of TPHA of $99 \%$, respectively. ${ }^{10,18}$

The results of this study were also different from the WHO evaluation in 2003 on six syphilis immunochromatographic tests which showed a sensitivity of $84.5 \%-97.7 \%$ and a specificity of 
92.8-98\%. ${ }^{21}$ Study by Herring et al. of nine syphilis immunochromatographic tests showed sensitivity and specificity of $84.5 \%-97.7 \%$ and $84.5-98 \%$, respectively. ${ }^{22} \mathrm{~A}$ study by Bazzo et al. on seven syphilis immunochromatographic tests showed a sensitivity and specificity of $94.5 \%-100 \%$ and 91.5\%-100\%. ${ }^{23}$ All of these studies used TPHA/TPPA as a confirmation test for treponemal antibody.

The syphilis immunochromatograpic test by WHO evaluation used one or more than one antigen combination. Two immunochromatographic tests using a combination of three antigens (Tp15, Tp17, and Tp47) showed a sensitivity and specificity of 95-97.7\% and 93.4-94.9\%, respectively. Two immunochromatographic tests using a combination of two antigens (Tp17 and Tp47) showed a sensitivity and specificity of $84.5-85 \%$ and $93.4-98 \%$, respectively. One immunochromatographic test using a combination of two antigens (Tp15 and Tp17) showed a sensitivity and specificity of $86 \%$ and $92.8 \%$, respectively. One immunochromatographic test using one antigen (Tp47) showed with a sensitivity and specificity of $97.2 \%$ and $94.1 \%$, respectively. ${ }^{21}$

The evaluation showed that the combination of three antigens had a better diagnostic value than the combination of two antigens. The use of one antigen Tp47 also showed an equivalent diagnostic value to that of combination of three antigens. This was in line with reference suggesting that Tp47 antigens were the most immunogenic and dominant protein, while Tp17 and Tp15 were detected in fewer levels, despite of their equally strong immunogenicity. ${ }^{24}$

Study by Miranda and Sato suggested that although Tp47 showed activity as a component of major antigens, combination of Tp17, Tp15, and Tp47 antigen was important for syphilis serological tests. ${ }^{24}$

None of the syphilis immunochromatographic tests in the study used Tp17 antigens in a single form. So far, researchers have not found any immunochromatographic test using one single Tp17 antigen and its diagnostic value compared with the TPHA or FTA-ABS.

The limitation of this study was no use of routine TPHA test as a confirmation test for treponemal antibody so that the results of the study were less compared to the use of other antigens on syphilis immunochromatographic tests. No Western Blot analysis was performed to determine the possible presence of a treponemal antibody other than anti-Tp17. Another limitation was no observation of clinical data from donors and there were no non-treponemal tests performed in all samples with reactive anti-treponemal results detected by FTA-ABS test to determine the stage and disease progression.

\section{CONCLUSION AND SUGGESTION}

Immunochromatographic tests using Tp17 antigen to detect treponemal antibody of blood donors showed a moderate and significant concordance to FTA-ABS with a sensitivity of $69.8 \%$ and a specificity of $81 \%$. The sensitivity which was not high in this study was thought to be due to the use of a single antigen (Tp17) while treponemal antibody is formed against three main antigens Tp15, Tp17, and Tp47. Another possibility was the decrease of anti-Tp17 IgG in donors after syphilis therapy and the difference in gold standard with other studies (FTA-ABS vs. TPHA).

It was recommended that further research use routine TPHA test as a confirmation test, Western Blot to determine the possibility of antibodies other than anti-Tp17, non-treponemal test such as VDRL or RPR in all blood samples with reactive treponemal antibody, and analyze clinical history of donor to determine the stage and progression of the disease.

\section{REFERENCES}

1. Mbanya $D$. Use of quality rapid diagnostic testing for safe blood transfusion in resource-limited settings. Clinical Microbiology and Infection. 2013; 19: 416-420

2. Morshed MG, Singh E. Recent trends in the serologic diagnosis of Syphilis. Clinical and Vaccine Immunology. 2015; 22(2): 137-147.

3. White BL, Sparling PF. Novel Treponema pallidum serologic tests $\square$ : A paradigm shift in syphilis screening for the $21^{\text {st }}$ century. Clinical Infectious Disease. 2018; 51(6): 700-708.

4. Pusat data dan informasi Kementerian Kesehatan RI. Situasi Pelayanan Darah di Indonesia. 2014. Diakses tanggal 3 April 2018.

5. Permenkes 83 Tahun 2014. Unit Transfusi Darah, Bank Darah Rumah Sakit, dan Jejaring Pelayanan Transfusi Darah. 2014. Diakses tanggal 25 April 2018.

6. Kubanov A, Runina A, Deryabi D. Novel Treponema pallidum recombinant antigens for syphilis diagnostics: Current status and future prospects. Hindawi BioMed Research International, 2017; 1-5.

7. Zhang R, Wang Q, Zhang J, Yang L. Tp17 membrane protein of Treponema pallidum activates endothelial cells in-vitro. International Immunopharmacology, 2015; 25: 538-544.

8. Sato NS, Suzuki T, Ueda T, Watanabe K, Hirata RD, Hirata $\mathrm{MH}$. Recombinant antigen-based immuno-slot blot method for serodiagnosis of syphilis. Brazilian 
Journal of Medical And Biological Reseach, 2004; 37(7): 949-955.

9. Simcic S, Potocnik M. Serological diagnosis of syphilis: A comparison of different diagnostic methods. Acta Dermatovenerologica, 2015; 24: 17-20.

10. Sena AC, White BL, Sparling PF. Novel Treponema pallidum serologic test: $A$ paradigm shift in syphilis screening for the $21^{\text {st }}$ century. Clinical Infectious Disease, 2010; 51(6): 700-708.

11. Soreng C, Levy R, Fakile Y. Serologic testing for syphilis: Benefits and challenges of a reverse algorithm. Clinical Microbiology Newsletter. Elsevier Inc. 2014; 36(24): 195-202.

12. EUROIMMUN AG. IgM \& IgG anti Treponema pallidum indirect immunofluorescent assay. EUROIMMUN, AG. 2014

13. Sambri V, Marangoni A, Eyer C, Reichhuber C, Soutschek E, Negosanti M, D'Antuono A, Cevenini R. Western immunoblotting with five Treponema pallidum recombinant antigens for serologic diagnosis of syphilis. Clinical and Diagnostic Laboratory Immunology. 2001; 534-539.

14. Lafond RE, Lukehart SA. Biological basis for syphilis. Clinical Microbiology Review. 2006; 19(1): 29-49.

15. Lin $L R$, Tong ML, Fu ZG, Dan B, Zheng WH, Zhang CG, Yang TC, Zhang YZ. Evaluation of colloidal gold immunochromatography assay in the detection of Treponema pallidum specific IgM antibody in syphilis serofast reaction patients: A serologic marker for the relapse and infection of syhilis. Diagnostic Microbiology and Infectious Disease. 2011; $70: 10-16$.

16. Sato NS. Laboratory diagnosis of syphilis. 2011; 89-92. www.intechopen.com. Access on $3^{\text {rd }}$ September, 2018
17. Ran S, Di-hui L, Rong-xin R, Shi L, Hai-ping Z. Treponema pallidum-specific antibody expression for the diagnosis of different stages of syphilis. Chin Med J. 2013; 126(2): 206-209.

18. Larsen SA, Steiner BM, Rudolph AH. Laboratory diagnosis and interpretation of tests for syphilis. Clinical Microbiology Review. 1995; 8(1): 1-21.

19. Adegoke AO, Akanni OE. Survival of Treponema pallidum in banked blood for prevention of syphilis transmission. North American Journal of Medical Science, 2011; 3(7): 329.

20. Orton S. Syphilis and blood donors: What we know, what we do not know, and what we need to know. Transfusion Medicine Review, 2001; 15(4): 282-292.

21. UNDP, WHO. Laboratory-based evaluation of rapid syphilis diagnostics. The sexually transmitted disease diagnostics initiative (SDI): Special program for reseach and training in tropical disease (TDR). 2003; 1 : 22-27.

22. Herring AJ, Ballard, RC., Pope, V., Adegbola, RA., Changalucha, J., Fitzgerald, DW., Hook, EW., et al. A multi-centre evaluation of nine rapid, point-of-care syphilis tests using archived sera. Sex Transm Infect, 2006; 82(V): 7-12.

23. Bazzo ML, da Motta LR, Rudolf-Oliveira RCM, Bigolin A, Golfetto L, Mesquita F, Benzaken AS, et al. Evaluation of seven rapid tests for syphilis available in Brazil using defibrinated plasma panels. Sex Transm Infect, 2017; 93: 46-50.

24. Miranda APF, Sato NS. Profile of anti-Tp47 antibodies in patients with positive serology for syphilis analized by western blot. Brazilian Journal of Infectious Diseases, 2008; 12(2): 139-143. 